\title{
Synthesis of porous graphene nanocomposite and its excellent adsorption behavior for Erythromycin antibiotic
}

\author{
Fateme Bahmei $^{1}$, Nader Bahramifar ${ }^{1 *}$, Habibollah Younesi ${ }^{1}$, Valeri Tolstoy ${ }^{2}$ \\ ${ }^{1}$ Department of Environmental Science, Faculty of Natural Resources, Tarbiat Modares University, Tehran, Iran \\ ${ }^{2}$ Department of Chemistry, Saint Petersburg State University, \\ Peterhof, 198504, Saint Petersburg, Russia \\ f.bahmei@modares.ac.ir, *n.bahramifar@modares.ac.ir, hunesi@modares.ac.ir, v.tolstoy@spbu.ru
}

\begin{abstract}
The purpose of this study was to evaluate the efficiency of porous magnetic graphene (PMG) for removal of Erythromycin (ER) from aqueous solutions. PMG was prepared from banana peel residue, which was considered as a discarded material. As-synthesized nanocomposite was characterized by SEM, AFM, FTIR, RAMAN and BET analysis. The optimum conditions were obtained at pH of 3 , contact time of 30 min, initial antibiotic concentration of $200 \mathrm{mg} / \mathrm{L}$, and adsorbent dose of $0.35 \mathrm{~g} / \mathrm{L}$. In equilibrium, the Langmuir isotherm model was the best fit to the experimental data for the kinetics study, the adsorption process developed the pseudo-second-order model. According to the results, nanosheet had high adsorption capacity ( $286 \mathrm{mg} / \mathrm{g}$ ) and can be considered as an acceptable adsorbent for the removal of ER from aqueous solutions.
\end{abstract}

Keywords: Porous magnetic graphene, Erythromycin, adsorption, kinetic.

Received: 3 March 2020

\section{Introduction}

Drugs, such as antibiotics are non-biodegradable. They can maintain their structure in the aquatic environment for a long time Erythromycin is one of the common types of antibiotics used for many infections. This antibiotic, like Erythromycin, is included in a macrolide antibiotic, so it can be used as a substitute for penicillin for patients who are allergic to beta-lactam antibiotics. Some antibiotics can't be removed through conventional wastewater treatment and they will reach surface water, groundwater and drinking water. Due to the necessity of eliminating antibiotics in the environment, there are various methods for removing them by researchers that each has its advantages and disadvantages [1]. Physical operations such as liquid phase extraction, adsorption, and membrane removal have been used to remove antibiotics. Biological processes, chemical processes such as electrochemistry, sound waves, chemical oxidation, and advanced oxidation have also been reported to be useful for antibiotic treatment. In physical methods, pollutants are generally separated from sewage or polluted by a physical mechanism and no change in the chemical structure of the contaminant. Therefore, these methods are more suitable for condensation as a pre-purification process [2].

Methods that are mainly effective in removing hormone and antibiotic compounds and, according to some evidence, other antibiotic compounds can be advanced techniques such as adsorption by activated carbon, reverse osmosis and oxidation by chlorine. The adsorption methods are simple and practical and have no toxic by-products and are low cost. One of them is Activated carbon that has high porosity and specific surface area and very high adsorption capacity that is widely used to remove organic pollutants from polluted waters and wastewater [3]. Graphene is another adsorbent that used to completely remove antibiotics from aqueous solutions. It is a new adsorbent in wastewater treatment [4]. In theory, it is not new, as it is the Fundamental structural building block of graphite and has been believed to occur for almost 100 years. Graphene sheets are made up of carbon atoms. On a graphene plate, each carbon atom is bonded to the other three carbon atoms. These three links are on one plate and the angles between them are equal to $120^{\circ}$. In this case, the carbon atoms are positioned to form a network of regular hexagons. The carbon-carbon bond length in graphene is about $0.142 \mathrm{~nm}$ [5].

Graphene is differed from other conventional 2D materials and has different structural properties. It is a terrific pure material that has unique and remarkable features due to it's simple and orderly structure (rising from the tight arrangement of carbon atoms), which can each make a significant revolution in the various fields of science and industry. Graphene is known as a transparent and flexible material [6]. Among the adsorbents, because of their easily of separation, magnetic adsorbents are more common. Therefore, the combination of nanotechnology and magnetic separation together has helped dramatically the complete elimination of antibiotics [7].

There are various methods of producing graphene. Such as reduction of graphene oxide [8,9], thermal decomposition of $6 \mathrm{HSiC}$ single crystal [10], chemical exfoliation, chemical vapor deposition (CVD), and other chemical 
synthesis [11]. In this investigation, porous magnetic graphene (PMG) synthesized naturally by using lignocellulosic biowastes such as banana peel, in addition to being a simple and cost-effective and straightforward, also introduces a little pollution into the environment. Then, the adsorption efficiency of as synthesized graphene were evaluated by removal of Erythromycin from synthetic wastewater. The parameters of solution $\mathrm{pH}$, temperature, adsorbent dosage, and primary concentration of Erythromycin which affect the omission process were explored.

\section{Experimental}

\subsection{Materials}

The Banana peel was used as a precursor for synthesis of graphene. Ferric chloride hexahydrate $\left(\mathrm{FeCl}_{3} \cdot 6 \mathrm{H}_{2} \mathrm{O}-\right.$ Merck), Sodium acetate $\left(\mathrm{C}_{2} \mathrm{H}_{3} \mathrm{NaO}_{2}\right.$-Merck), Potassium hydroxide( $\left.\mathrm{KOH}-\mathrm{Merck}\right)$, Ethylene glycol $\left(\mathrm{C}_{2} \mathrm{H}_{6} \mathrm{O}_{2}-\mathrm{Merck}\right)$, and Ethanol $\left(\mathrm{C}_{2} \mathrm{H}_{5} \mathrm{OH}-\right.$ Merck) were used in the synthesis of PMG. Erythromycin $\left(\mathrm{C}_{37} \mathrm{H}_{67} \mathrm{NO}_{13}\right)$ used in the current study was purchased from sigma.

\subsection{Synthesis of graphene}

For the preparation of graphene, Banana peels were first crushed and then have been washed well to remove pollutants. Then they were dried in an oven at $70{ }^{\circ} \mathrm{C}$. To obtain the uniform particle size, it has been milled and passed from the sieve $10 \mathrm{~g}$ of the dried material was carbonated in a muffle furnace at a temperature of $600{ }^{\circ} \mathrm{C}$ with a rate of $10^{\circ} \mathrm{C} \mathrm{m^{-1 }}$ with the attendance of argon gas for 1 hour. $5 \mathrm{~g}$ of carbonized sample was mixed with $25 \mathrm{~g} \mathrm{KOH} \mathrm{(1:5}$ ratio) and stirred for 1 hour on a magnetic stirrer. Then it will dry for 24 hours (at $110^{\circ} \mathrm{C}$ ). To get the graphene, the activated sample was moved to the furnace and heated at $900{ }^{\circ} \mathrm{C}$ for 2 hours at a temperature rate of $10{ }^{\circ} \mathrm{C} \mathrm{min}{ }^{-1}$. Then, the sample will cool in the presence of argon gas flow. To remove extant potassium, sample was leach with distilled water until the $\mathrm{pH}$ of the washed water became 6 to 7 . Finally, the sample was dried in an oven at $110^{\circ} \mathrm{C}$ for 24 hours. The pictorial representation of the procurement of graphene is given in Fig. 1.
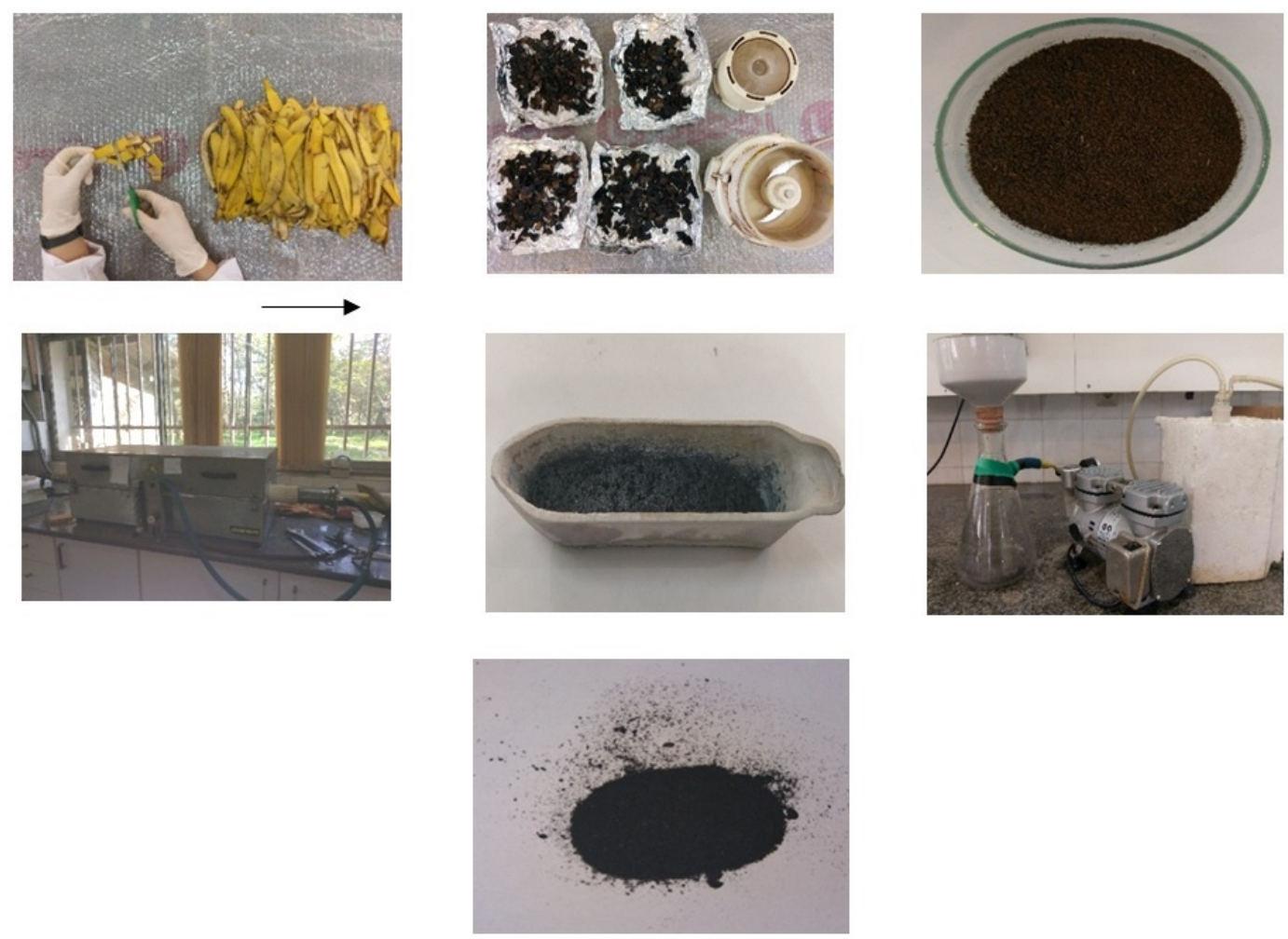

FIG. 1. Process of graphene preparation 


\subsection{Synthesis of PMG}

Graphene $(0.5 \mathrm{~g}$ ) was exfoliated in $70 \mathrm{ml}$ ethyleneglycol (EG) for $3 \mathrm{~h}$ using ultrasonic. Ferric chloride hexahydrate $(1 / 5 \mathrm{~g})$ and sodium acetate $(3 \mathrm{~g})$ was added to the above mixture and stirred at $500 \mathrm{rpm}$ for 30 minutes. Next the mixture was transferred into a $100 \mathrm{~mL}$ Teflon-lined stainless-steel autoclave and kept at $200{ }^{\circ} \mathrm{C}$ for $10 \mathrm{~h}$. The sample was washed with distilled water and ethanol (4 to 6 steps), and finally were vacuumed at $60^{\circ} \mathrm{C}$ for $6 \mathrm{~h}$ until complete drying [12].

\section{Characterization methods}

G and PMG samples were defined by X-ray diffraction (XRD) measurements using a Philips X'PERT MPD. The Fourier Transform Infrared spectroscopy (FTIR) of the samples was obtain using a Shimadzu FT-IR spectrophotometer in the range of $400-4000 \mathrm{~cm}^{-1}$. The measurement and preparation method is KBr Pellet Method. VSM measurements were carried out by using a Vibrating Sample Magnetometer (Meghnatis Daghigh Kavir Co. Kashan, Iran). Raman spectra were taken with Raman Spectrometer Teksan P50C0R. Raman spectrometers use LASER wavelengths of 533 and $633 \mathrm{~nm}$ during characterizing Graphene. Specific surface area values of samples were calculated by the BrunauerEmmett-Teller (BET) method. The morphology and microstructure of the samples were observed using a scanning electron microscope (SEM) (LEO 1455 VP, Cambridge, U.K.). Atomic force microscope (AFM) image was taken by AFM- Park Scientific CP-Research model (VEECO).

\section{Result and discussion}

\subsection{Characterization of the adsorbent}

AFM is a powerful tool for measuring sample thickness. The height profile of the synthesized G sheet from banana peels is presented in AFM images of Fig. 2. From AFM cross-section analysis, it can be observed that the synthesized graphene possesses thickness in the range of 1 to $2.8 \mathrm{~nm}$ [13].
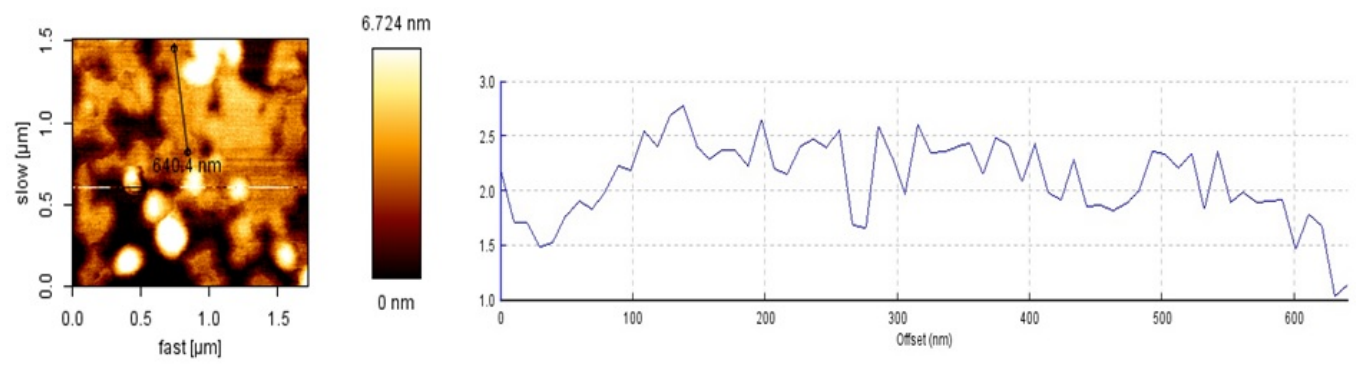

FIG. 2. The AFM images of graphene and its cross-section analysis

Raman spectroscopy is one of the most potent experiments to identify carbon derivatives such as graphene. Raman spectroscopy is an effective method to show the properties of single and multilayer graphene. Graphene structure can be determined by examining the location and intensity of the peaks. The Raman spectrum obtained from the graphene was shown in Fig. 3.

The Raman spectrum shows a fundamental vibrational band in the range between $1300-1700 \mathrm{~cm}^{-1}$. The two significant groups are introduced as $\mathrm{G}$ and 2D bands. Besides, a third band will also appear if there is a defect in the carbon network in the graphene ( $\mathrm{D}$ band). The 2D band of the Raman spectrum has been more sensitive to graphene sheets accumulation [14]. The 2D band position of the graphene monolayer is $2670 \mathrm{~cm}^{-1}$, whereas for several graphene layers (containing 2-4 layers), the 2D band position shifts to larger numbers. The proportion of $\frac{\mathrm{I}_{2 D}}{\mathrm{I}_{G}}$ for single-layer graphene without defects will be 1/6-2. In this study, 2D band at $2619 \mathrm{~cm}^{-1}$, indicating the presence of two- and three-layer graphene sheets in the as-synthesized graphene $[15,16]$.

The outcomes of the FTIR spectrum of synthesized graphene and PMG nanocomposite are shown in Fig. 4. The functional groups -OH (at nearly $3436 \mathrm{~cm}^{-1}$ ), C-H (between $2848-2923 \mathrm{~cm}^{-1}$ ) C=C (about $1535 \mathrm{~cm}^{-1}$ ), and C-O-C groups (about $1050 \mathrm{~cm}^{-1}$ ) perceived on the surface for both graphene and magnetic graphene. FTIR spectrum of Magnetic graphene gives new bands at $593 \mathrm{~cm}^{-1}$, which can be attribute to $\mathrm{Fe}-\mathrm{O}$ bond and provides the witness with successful procurement of magnetic graphene [17, 18].

SEM is predominantly used to study the morphology of the synthesized sample and to interpret the results of the synthesis on a micron or nanometer scale. Fig. 5(a) shows the synthesized graphene. The SEM image of graphene 


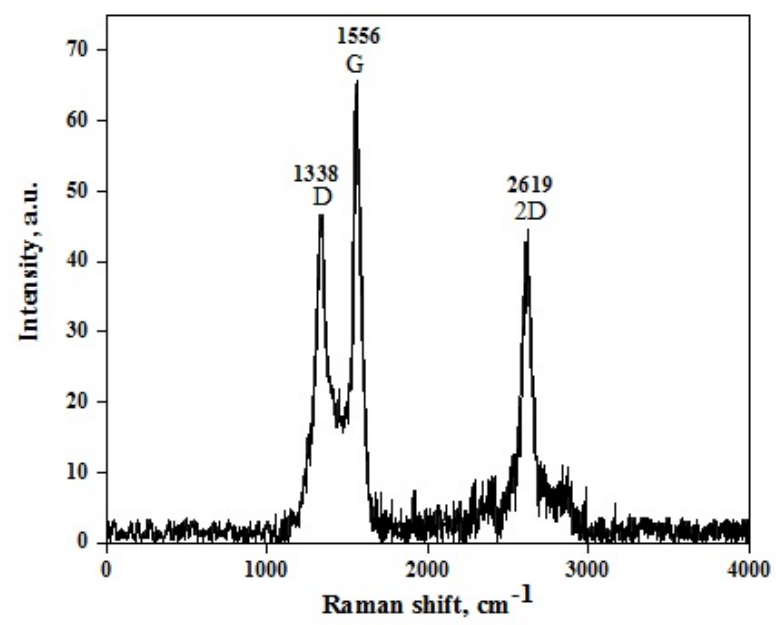

FIG. 3. Raman spectrum from the synthesized graphene

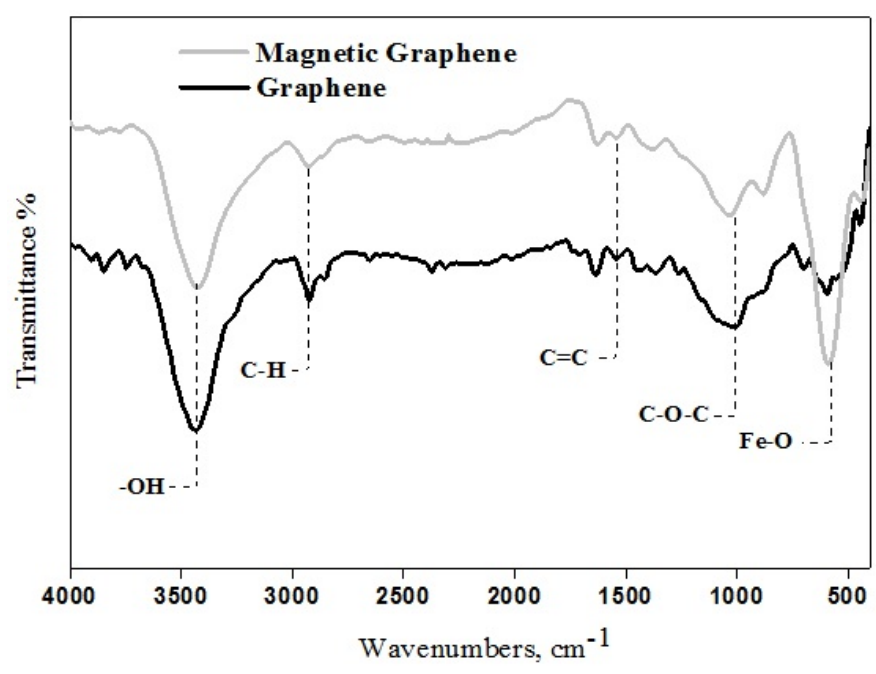

FIG. 4. The FTIR spectrum of synthesized graphene and magnetic graphene

illustrates the few layered structure. Fig. 5(b) shows spherical $\mathrm{Fe}_{3} \mathrm{O}_{4}$ nanoparticles that are well located on graphene layers and have relatively uniform sizes between 50 and $100 \mathrm{~nm}$ [19].
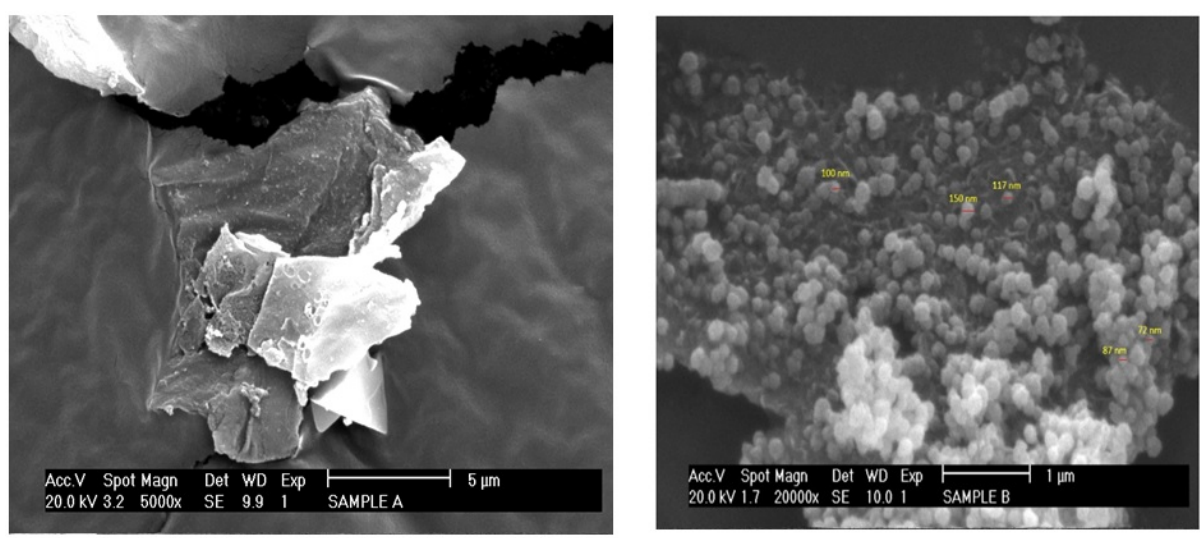

FIG. 5. SEM images of the graphene (a) and magnetic graphene(b) 
One of the most critical methods for accurately measuring the total area of porous samples is the BET method, which is based on the adsorption of individual molecular species in the gas state on their surface. From Fig. 6, the significant consequence of the activation parameters in the progression of the porosity by $\mathrm{N}_{2}$ isotherms shows a type I isotherm for $\mathrm{N}_{2}$ adsorption on graphene according to the IUPAC classification. It indicates the characteristic of a mainly microporous material. The BJH (Barrett, Joyner, and Halenda) and MP (Microporisity Plot) diagrams showed that the graphene and PMG synthesized mostly contained micro-spherical structures (Fig.7). The percentage of microcavity structures in graphene was $96 \%$ and for magnetic graphene $87 \%$. In previous studies, the theoretical specific surface area of graphene has been expressed as approximately $2630 \mathrm{~m}^{2} \mathrm{~g}^{-1}$. The specific surface area of synthesized graphene is obtained at $2317.5 \mathrm{~m}^{2} \mathrm{~g}^{-1}$ and is very close to the theoretical value. In addition to the specific surface area of synthesized graphene, other features such as micropore volume $\left(\mathrm{V}_{\text {mic }}\right)$, mesopore volume $\left(\mathrm{V}_{\text {mes }}\right)$, total volume $\left(\mathrm{V}_{\text {total }}\right)$ and cavity radius $(\mathrm{R})$ are given in Table 1 . The results show that both graphene and porous magnetic graphene mainly comprise microporous structures [11].

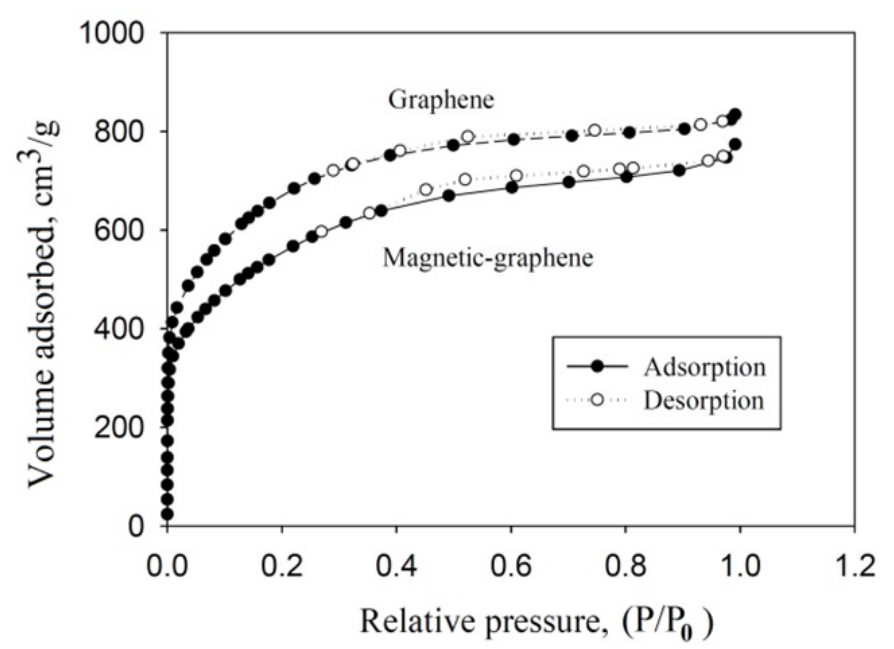

FIG. 6. Nitrogen Adsorption-desorption isotherm of graphene and PMG

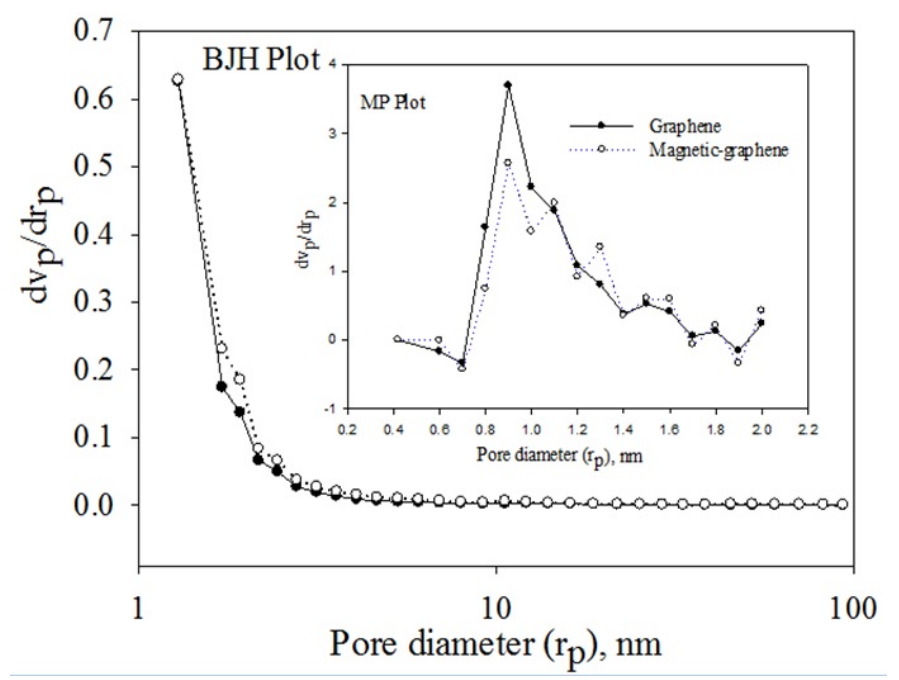

FIG. 7. Pore size distribution of graphene and PMG by using MP and BJH method

\subsection{Adsorption experiments}

The ER adsorption virtues of PMG were studying at initial $\mathrm{pH}$ values between 3 and 9 (Fig. 8). When the pH value is 3, the adsorption capacity of PMG is high. At $\mathrm{pH}$ higher than 3 the ER molecule has been negative charge and its tendency to adsorb onto adsorbent has been decreased. The optimal $\mathrm{pH}$ value was considered to be 5 . In 
TABLE 1. Structural properties of the graphene and PMG

\begin{tabular}{|c|c|c|c|c|c|}
\hline Sample & $\mathrm{S}_{B E T}, \mathrm{~m}^{2} \mathrm{~g}^{-1}$ & $\mathrm{~V}_{m i c}, \mathrm{~cm}^{3} \mathrm{~g}^{-1}$ & $\mathrm{~V}_{\text {mes }}, \mathrm{cm}^{3} \mathrm{~g}^{-1}$ & $\mathrm{~V}_{\text {total }}, \mathrm{cm}^{3} \mathrm{~g}^{-1}$ & $\mathrm{R}, \mathrm{nm}$ \\
\hline Graphene & 2317.5 & 1.23 & 0.05 & 1.28 & 1.29 \\
\hline Magnetic Graphene & 1905.5 & 1.05 & 0.14 & 1.19 & 1.29 \\
\hline
\end{tabular}

this $\mathrm{pH}$ range, the percentage of antibiotic removal was 55\%, respective [20]. The effect of reaction time is shown in Fig. 8(a). In the present study, as the contact time increased, the adsorption capacity was increased due to the increased probability of the antibiotic molecule being used to adsorb surface. The adsorption of antibiotic was very rapid in the early minutes and decreased rapidly over time, which may be due to the filling of the adsorbent pores or the problematic access of the antibiotic molecules to the active sites on the adsorbent surface. The rapid adsorption of antibiotics at the initial contact time can be assigned to the high surface area of PMG. The equilibrium contact time of the antibiotic adsorption was 30 minutes, with the elimination rate at this time being approximately $83 \%$ for Erythromycin (Fig. 8(b)). [21]. The results of adsorbent dosage on the removal percentage and equilibrium adsorption capacity of the antibiotic molecules studied are presented in Fig. 8(c). As shown in the figure, the percentage of ER removal increased from 32 to 85 as the adsorbent increased. The optimum dose of adsorbent was $35 \mathrm{mg} / \mathrm{L}$. As shown in Fig. 2(d), with increasing concentration, the equilibrium adsorption capacity for ER increased. The maximum absorption capacity of Erythromycin was $286 \mathrm{mg} / \mathrm{g}$ at $200 \mathrm{mg} / \mathrm{L}$ ER concentration. The results show, as the initial concentration of the contaminant increased, the adsorption efficiency decreased [22].

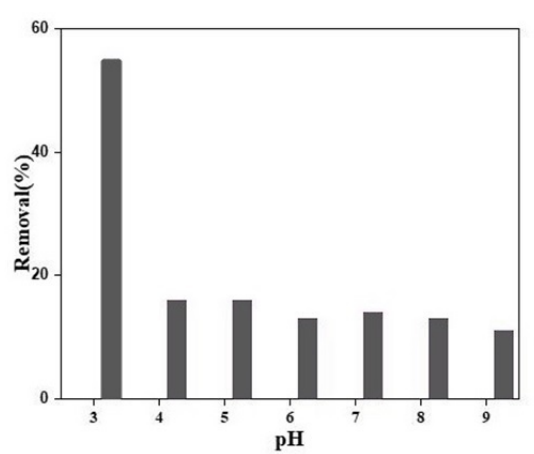

(a)

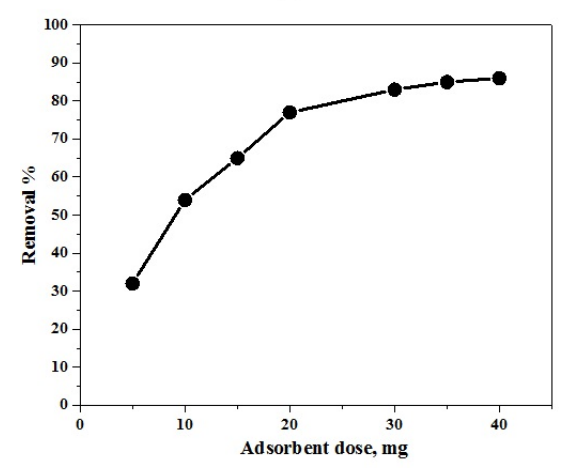

(c)

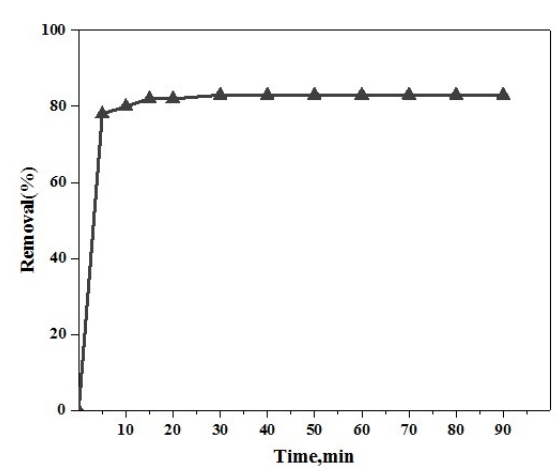

(b)

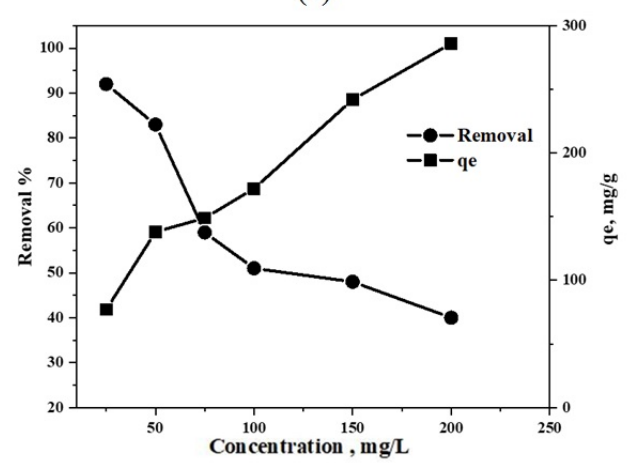

(d)

FIG. 8. (a) Effect of $\mathrm{pH}$ on the removal of ER by using adsorbent dosage $=20 \mathrm{mg}$, initial concentration $=50 \mathrm{mg} / \mathrm{L}$, at $25^{\circ}$, (b) Effect of contact time on the removal of ER by using $\mathrm{pH}=3$, adsorbent dosage $=30 \mathrm{mg}$, initial concentration $50 \mathrm{mg} / \mathrm{L}$, at $25^{\circ}$, (c) Effect of adsorbent dosage of PMG on the removal of ER by using $\mathrm{pH}=3$, initial concentration $50 \mathrm{mg} / \mathrm{L}$, at $25^{\circ}$, (d) Effect of the initial concentration of ER on removal efficiency and equilibrium adsorption capacity by using $\mathrm{pH}=3$, adsorbent dosage $=30 \mathrm{mg}$, at $25^{\circ}$

\subsection{Adsorption kinetics}

To discern the dynamics of the adsorption reaction, data can be explained by the adsorption kinetics. In the current study, to obtain these purposes, the Pseudo-first-order (Eq.1) and pseudo-second-order (Eq.2) kinetics model 
were investigated. According to the achieved results, the mean correlation coefficient $\left(\mathrm{R}^{2}\right)$ in the 2 -nd order kinetic model was higher than that of the 1st-order kinetic model. Consequently, the experimental data demonstrate that the adsorption of ER on PMG fitted well the 2nd-order kinetic model (Fig. 9(a,b)):

$$
\begin{gathered}
\log \left(q_{e}-q_{t}\right)=\log q_{e}-\frac{k_{1}}{2.303} t \\
\frac{t}{q_{t}}=\frac{1}{k_{2} q_{e}^{2}}+\frac{t}{q_{e}}
\end{gathered}
$$

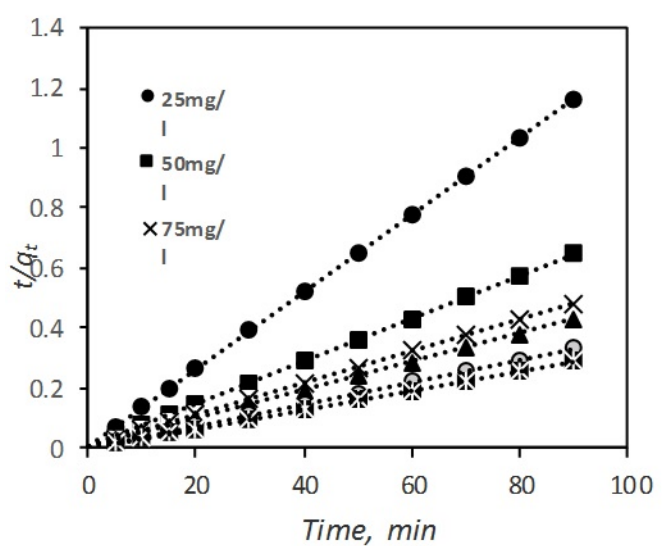

(a)

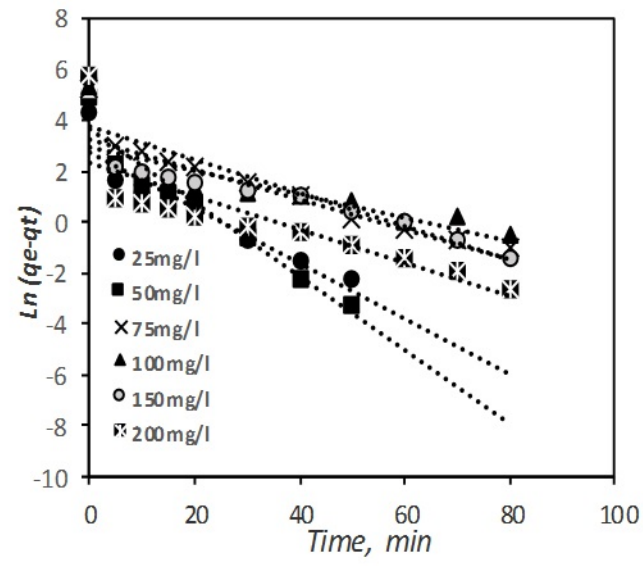

(b)

FIG. 9. Adsorption kinetics of ER onto PMG: (a) pseudo-first-order, (b) pseudo-second-order model

The relevant kinetic parameters calculatedand are listed in Table 2. The results show that the calculated adsorption capacity $\left(\mathrm{qe}_{2}\right)$ of the pseudo-second order model for ER absorption is closer to the experimental adsorption capacity [23, 24].

TABLE 2. Kinetic parameters for pseudo-first-order and pseudo-second-order

\begin{tabular}{|c|c|c|c|c|c|c|c|}
\hline \multicolumn{2}{|c|}{ ER } & \multicolumn{3}{c|}{ Pseudo-first-order } & \multicolumn{3}{c|}{ Pseudo-second-order } \\
\hline Concentration(mg/L) & $\mathrm{q}_{e}^{\text {exp }}(\mathrm{mg} / \mathrm{g})$ & $\mathrm{k}_{1}\left(\mathrm{~min}^{-1}\right)$ & $\mathrm{q}_{e 1}(\mathrm{mg} / \mathrm{g})$ & $\mathrm{R}^{2}$ & $\mathrm{k}_{2}\left(\mathrm{~g} \mathrm{mg}^{-1} \mathrm{~min}^{-1}\right)$ & $\mathrm{q}_{e 2}(\mathrm{mg} / \mathrm{g})$ & $\mathrm{R}^{2}$ \\
\hline 25 & 77.24 & 0.109 & 15.98 & 0.8712 & 0.031 & 77.52 & 0.9999 \\
50 & 138.80 & 0.143 & 36.75 & 0.9394 & 0.001 & 129.87 & 0.9999 \\
75 & 187.01 & 0.065 & 42.74 & 0.9113 & 0.005 & 188.67 & 0.9999 \\
100 & 210.46 & 0.045 & 18.57 & 0.6571 & 0.011 & 212.77 & 0.9999 \\
150 & 272.44 & 0.059 & 26.41 & 0.7758 & 0.010 & 270.27 & 0.9999 \\
200 & 310.44 & 0.065 & 10.42 & 0.6785 & 0.034 & 312.52 & 0.9999 \\
\hline
\end{tabular}

\subsection{Adsorption isotherms}

To study the adsorbate distribution over solid/liquid boundaries, the adsorption isotherm is the basic tool [25] In the current study, commonadsorption isotherm models such as Langmuir, Freundlich and Sips were used to obtain detailed information about the interactions between the surface of the PMG and ER. The equations of these models are 3, 4 and 5 Equations:

$$
\begin{gathered}
q_{e}=\frac{q_{m}+b C_{e}}{1+b C_{e}} \\
q_{e}=k_{f} C_{e}^{1 / n} \\
q_{e}=\frac{Q_{L} K_{L} C_{e}^{n_{s}}}{1+k_{s} C_{e}^{n_{s}}}
\end{gathered}
$$


where, $C_{e}$ and $q_{e}$ represent the equilibrium concentration in the soluble $(\mathrm{mg} / \mathrm{l})$ and solid $(\mathrm{mg} / \mathrm{g}), q_{m}$ showed maximum adsorption capacity $(\mathrm{mg} / \mathrm{g})$ and $\mathrm{b}, \mathrm{n}$ and $\mathrm{k}$ parameters are isotherms constants Fig. 10 demonstrates the plots of adsorption isotherm models and isotherm parameters are shown in Table 3 [26].

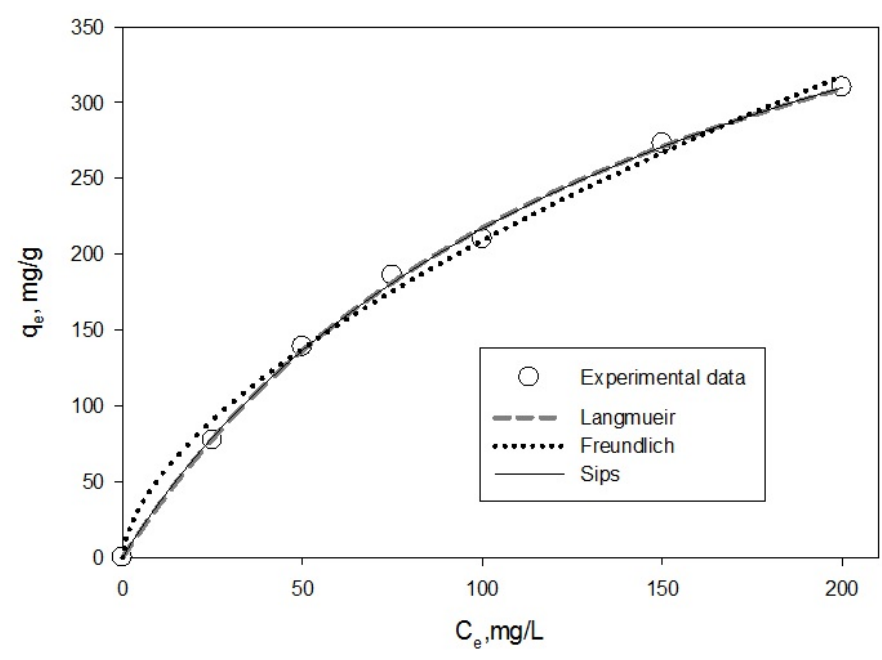

FIG. 10. Adsorption isotherms of ER onto PMG

By comparing the correlation coefficients among isotherm models, it was found that the Langmuir isotherm could describe the experimental data better than the other models. This result showed that the process is homogeneous and monolayer adsorption [27].

TABLE 3. Langmuir, Freundlich and SIPS adsorption isotherm fitting parameters of ER on PMG

\begin{tabular}{|c|c|c|c|}
\hline Adsorption isotherm & Parameter & Value & R2 \\
\hline \multirow{2}{*}{ Langmuir } & $\mathrm{q}_{m}$ & 534 & \multirow{2}{*}{0.9973} \\
\cline { 2 - 3 } & $\mathrm{b}_{(l . m g)}$ & 0.0005 & \\
\hline \multirow{2}{*}{ Freundlich } & $\mathrm{K}_{F}$ & 13.03 & \multirow{2}{*}{0.9798} \\
\cline { 2 - 3 } & $\mathrm{n}$ & 1.65 & \\
\hline \multirow{3}{*}{ Sips } & $\mathrm{q}_{m}$ & 561 & \multirow{2}{*}{0.7291} \\
\cline { 2 - 3 } & $\mathrm{K}_{s}$ & 0.0062 & \\
\cline { 2 - 3 } & $\mathrm{n}$ & 1.03 & \\
\hline
\end{tabular}

\subsection{Conclusions}

The primary objective of this study was to synthesize high-quality graphene from the banana peel with low chemical consumption For better separation from aqueous solution, porous magnetic graphene was synthesized. Then, it was confirmed by AFM, Raman, FTIR, SEM, and BET analyses Based on the morphological results, the porous surface was confirmedand it is demonstrated that suggested adsorbent has the ability to remove antibiotic. The optimum condition was provided at $\mathrm{pH}$ of 3 , contact time of $30 \mathrm{~min}$, and the adsorbent dose of $0.35 \mathrm{~g} / \mathrm{L}$. Also, the results exposed that the Langmuir isotherm and pseudosecond-order kinetics model better describes the adsorption of Erythromycin. According to the results, PMG nanoparticle adsorbent can be considered as an acceptable absorbent for the removal of antibiotics from aqueous solutions.

\section{References}

[1] Li MF, Liu YG, Zeng GM, Liu N, Liu SB. Graphene and graphene-based nanocomposites used for antibiotics removal in water treatment: a review. Chemosphere, 2019.

[2] Koyuncu I., Arikan O.A., Wiesner M.R., Rice C. Removal of hormones and antibiotics by nanofiltration membranes. Journal of membrane science, 2008, 309(1-2), P. 94-101.

[3] Moussavi G., Allahabad A., Yaghmaeian K., Eskandari M. Preparation, characterization and adsorption potential of the $\mathrm{NH}_{4} \mathrm{Cl}$-induced activated carbon for the removal of amoxicillin antibiotic from water. Chemical engineering journal, 2013, 217, P. 119-128. 
[4] Yi J.W., Park J., Kim K.S., Kim B.H. pH-Responsive self-duplex of Py A-substituted oligodeoxyadenylate in graphene oxide solution as a molecular switch. Organic and biomolecular chemistry, 2011, 9(21), P. 7434-7438.

[5] Dhinakaran V., Lavanya M., Vigneswari K., Ravichandran M., Vijayakumar M.D. Review on exploration of graphene in diverse applications and its future horizon. Materials Today: Proceedings, 2020.

[6] Yousefi N., Lu X., Elimelech M., Tufenkji N. Environmental performance of graphene-based 3D macrostructures. Nature nanotechnology, 2019, 14(2), P. 107-119.

[7] Zhu J., Wei S., Gu H., Rapole S.B., Wang Q., Luo Z., Haldolaarachchige N., Young D.P., Guo Z. One-pot synthesis of magnetic graphene nanocomposites decorated with core@ double-shell nanoparticles for fast chromium removal. Environmental science and technology, 2012, 46(2), P. 977-985.

[8] Dideikin A.T., Vul A.Y. Graphene oxide and derivatives: the place in graphene family. Frontiers in Physics, 2019,6, P. 149.

[9] Shanmugam S., Nanjan S. In-situ conversion of rgo from graphene oxide based on solar mediated enhanced characterization properties. Nanosystems: Physics, Chemistry, Mathematics, 2019, 10(5), P. 579-584.

[10] Lebedev S.P., Davydov V.Y., Usachov D.Y., Smirnov A.N., Levitskii V.S., Eliseyev I.A., Guschina E.V., Dunaevsckiy M.S., Vilkov O.Y., Rybkin A.G., Lebedev A.A. Graphene on silicon carbide as a basis for gasand biosensor applications. Nanosystems: Physics, Chemistry, Mathematics, 2018, 9(1), P. 95-97.

[11] Ekhlasi L., Younesi H., Rashidi A., Bahramifar N. Populus wood biomass-derived graphene for high $\mathrm{CO}_{2}$ capture at atmospheric pressure and estimated cost of production. Process Safety and Environmental Protection, 2018, 113, P. 97-108.

[12] Ai L., Zhang C., Chen Z. Removal of methylene blue from aqueous solution by a solvothermal-synthesized graphene/magnetite composite. Journal of hazardous materials, 2011, 15, 192(3), P. 1515-1524.

[13] Lin L.Y., Kim D.E., Kim W.K., Jun S.C. Friction and wear characteristics of multi-layer graphene films investigated by atomic force microscopy. Surface and Coatings Technology, 2011, 205(20), P. 4864-4869.

[14] Villar-Rodil S., Paredes J.I., Martínez-Alonso A., Tascón J.M. Preparation of graphene dispersions and graphene-polymer composites in organic media. Journal of Materials Chemistry, 2009, 19(22), P. 3591-3593.

[15] Calizo I., Balandin A.A., Bao W., Miao F., Lau C.N. Temperature dependence of the Raman spectra of graphene and graphene multilayers. Nano letters, 2007, 7(9), P. 2645-2649.

[16] Ferrari A.C. Raman spectroscopy of graphene and graphite: disorder, electron-phonon coupling, doping and nonadiabatic effects. Solid state communications, 2007, 143(1-2), P. 47-57.

[17] Xue B., Zhu J., Liu N., Li Y. Facile functionalization of graphene oxide with ethylenediamine as a solid base catalyst for Knoevenagel condensation reaction. Catalysis Communications, 2015, 64, P. 105-109.

[18] Lü M., Li J., Yang X., Zhang C., Yang J., Hu H., Wang X. Applications of graphene-based materials in environmental protection and detection. Chinese Science Bulletin, 2013, 58(22), P. 2698-710.

[19] Guo X., Du B., Wei Q., Yang J., Hu L., Yan L., Xu W. Synthesis of amino functionalized magnetic graphenes composite material and its application to remove $\mathrm{Cr}$ (VI), $\mathrm{Pb}$ (II), $\mathrm{Hg}$ (II), Cd (II) and $\mathrm{Ni}$ (II) from contaminated water. Journal of hazardous materials, 2014, 278, P. 211-20.

[20] Ou H., Chen Q., Pan J., Zhang Y., Huang Y., Qi X. Selective removal of erythromycin by magnetic imprinted polymers synthesized from chitosan-stabilized Pickering emulsion. Journal of hazardous materials, 2015, 289, P. 28-37.

[21] Moussavi G., Alahabadi A., Yaghmaeian K., Eskandari M. Preparation, characterization and adsorption potential of the $\mathrm{NH}_{4} \mathrm{Cl}-\mathrm{induced}$ activated carbon for the removal of amoxicillin antibiotic from water. Chemical engineering journal, 2013, 217, P. 119-28.

[22] Budyanto S., Soedjono S., Irawaty W., Indraswati N. Studies of Adsorption Equilibria and Kinetics of amoxicillin from simulated wastewater using activated carbon and natural bentonite. Journal of environmental protection science, 2008, 2, P. 72-80.

[23] Shafiee M., Akbari A., Ghiassimehr B. Removal of Pb (II) From Wastewater Using Henna; Optimization of Operational Conditions. Iranian Journal of Chemical Engineering, 2018, 15(4), P. 17-26.

[24] Peer F.E., Bahramifar N., Younesi H. Removal of Cd (II), Pb (II) and Cu (II) ions from aqueous solution by polyamidoamine dendrimer grafted magnetic graphene oxide nanosheets. Journal of the Taiwan Institute of Chemical Engineers, 2018, 87, P. 225-40.

[25] Beltrame K.K., Cazetta A.L., de Souza P.S., Spessato L., Silva T.L., Almeida V.C. Adsorption of caffeine on mesoporous activated carbon fibers prepared from pineapple plant leaves. Ecotoxicology and environmental safety. 2018. 147, P. 64-71.

[26] Rostamian R., Behnejad H. A comparative adsorption study of sulfamethoxazole onto graphene and graphene oxide nanosheets through equilibrium, kinetic and thermodynamic modeling. Process Safety and Environmental Protection, 2016, 102, P. $20-9$.

[27] Budyanto S., Soedjono S., Irawaty W., Indraswati N. Studies of Adsorption Equilibria and Kinetics of amoxicillin from simulated wastewater using activated carbon and natural bentonite. Journal of environmental protection science, 2008, 2, P. 72-80. 